\title{
The Relation Between the Energy of a Hydrogen Bond and the Frequencies of the $\mathrm{O}-\mathrm{H}$ Bands*
}

\author{
RICHARD M. BADGER \\ Gates and Crellin Laboratories of Chemistry, California Institute of Technology, Pasadena, California
}

(Received November 6, 1939)

$I^{\mathrm{T}}$ has previously been pointed out ${ }^{1}$ that there appears to be a relation between the energy of a hydrogen bond and the shift in frequency of the $\mathrm{O}-\mathrm{H}$ bands which accompanies the formation of the bond. Recently additional data have been obtained in this laboratory ${ }^{2,3}$ which confirm the existence of this useful relation and it seems worth while to discuss them in this connection.

The data here presented all relate to linkages in which a hydroxyl hydrogen is concerned, though the atom to which it is weakly bound may be oxygen, chlorine, or carbon in an aromatic ring. It is surprising that the same connection between energy and frequency shift should be found in these various cases but such appears to be the fact. For other types of bond, for example those involving the hydrogen of an amino group, a slightly different relation may well be expected.

The data plotted in Fig. 1, with one exception, relate to cases in which a hydroxyl group which is initially in a relatively ideal, unperturbed condition enters into a hydrogen bond. The observed shift in frequency of the third harmonic $\mathrm{O}-\mathrm{H}$ band which accompanies this change is plotted against the energy of the bond. ${ }^{4}$ There is a fairly strong indication that the relation between frequency and energy is not linear, though the curve drawn is indeed somewhat arbitrary. The inaccuracy in the energy values may be at least as great as $\mathbf{5 0 0} \mathrm{cal}$. and the precision of the frequency measurements is uncertain. The frequencies employed are those of the band maxima. This is sa tisfactory in the case of narrow bands but the association bands are broad and unsymmetrical and it is not certain to what point one should measure. It seems quite

\footnotetext{
* Contribution No. 744 from the Gates and Crellin Laboratories of Chemistry.

1 Badger and Bauer, J. Chem. Phys. 5, 839 (1937).

${ }^{2}$ Lassettre and Dickinson, J. Am. Chem. Soc. 61, 54 (1939).

${ }^{3}$ Zumwalt and Badger, J. Am. Chem. Soc. (in press).

4 The data for water and methyl alcohol are those of reference 1
}

certain that resolvable features which these bands have sometimes been supposed to possess are merely superposed $\mathrm{C}-\mathrm{H}$ bands or other harmonic or combination bands. At present the maximum seems to be the least arbitrary point of reference for measurement.

The abscissae of the points for 0 -chloro phenol and ethylene chlorohydrin are differences in energy of two molecular configurations in one of which an intramolecular hydrogen bond is present. The energy difference of the two states may be partly due to other factors beside the hydrogen bond, but at present it is not possible to make allowance for them. Similar difficulties, however, are encountered in the estimation of the energy of any hydrogen bond. For example, in obtaining the energy of the two bonds in the dimer organic acids from the dissociation energy one should probably make some allowance for van der Waals interactions.

It is not certain that it is permissible to include the point for phenol in benzol solution in the diagram. The spectroscopic data ${ }^{5}$ indicate a very low $\mathrm{O}-\mathrm{H}$ frequency for the phenol monomer in

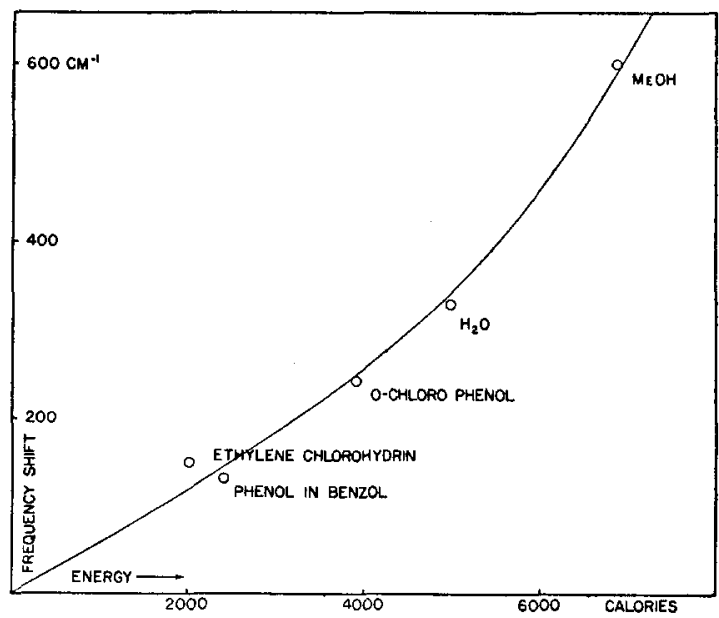

Fig. 1. A plot of the frequency shift in the third harmonic $\mathrm{O}-\mathrm{H}$ bands against energy of the hydrogen bond.

${ }^{5}$ A. Naherniac, Ann. de physique 7, 528 (1937). 
benzol solution, which may be taken as evidence for solvation through the agency of a hydrogen bond. The energy quantity evaluated by Lassettre and Dickinson ${ }^{2}$ may thus be regarded as the difference in energy of two hydrogen bonds. If the energy frequency relation is nonlinear one should expect the point for phenol to lie somewhat above the curve fitting the other points. A similar situation may be expected for the carboxylic acids. The low $\mathrm{O}-\mathrm{H}$ frequency and the rather rigid planar $\mathrm{COOH}$ group of the acid monomers ${ }^{6}$ suggest some tendency for the formation of a weak hydrogen bond in a four-membered ring.

The frequency shifts plotted in the figure are all for the third harmonic $\mathrm{O}-\mathrm{H}$ bands in the region $\lambda 9500-11,000$. The photograph region, beside being convenient for investigation, has the advantage that shifted and unshifted bands are better separated and more accurately measured than in the fundamental region. It would no doubt be preferable to deal with frequencies extrapolated to zero amplitude but the data are at present too incomplete to permit this. In any case the frequency shifts in the third harmonic bands will usually be nearly proportional to

\footnotetext{
${ }^{6}$ Bauer and Badger, J. Chem. Phys. 5, 852 (1937).
}

those in the $\omega_{e}$ 's, since the convergence of most $\mathrm{O}-\mathrm{H}$ band progressions is about the same.

A very important exception to this is the case of the carboxylic acids. By a somewhat unjustifiable method of plotting Badger and Bauer brought a point for acetic acid in line with other data, but actually the bands of the associated organic acids are quite anomalous. Badger and Bauer have discussed the character of the $\mathrm{O}-\mathrm{H}$ vibrations in these substances and the nature of the potential function. As they pointed out, an unsymmetrical $\mathrm{O}-\mathrm{H}$ vibration is responsible for the infra-red association bands. Owing to the somewhat special character of the potential function the succession of energy levels corresponding to this vibration must diverge rather than converge, as is customary. One is consequently not surprised to find that the progression of $\mathrm{O}-\mathrm{H}$ bands for the double molecules diverges at about the same rate as other $\mathrm{O}-\mathrm{H}$ progressions converge. Such anomalous cases are probably rare.

Many applications of the relations here discussed must be obvious and need not be discussed. Recently spectroscopic data relating to hydrogen bonds have been accumulating rapidly and in many cases they may greatly assist in interpreting observations of other kinds. 